\title{
HERRAMIENTAS DE TECNOLOGÍA DE GESTIÓN EN LIMA METROPOLITANA
}

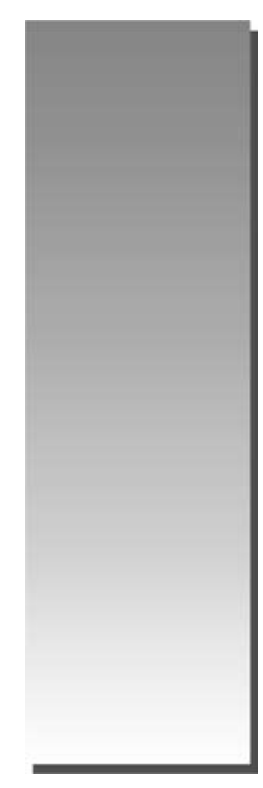

Enrique Huaco Pastor (*)

E-mail: enrique_huaco@yahoo.com

\begin{abstract}
RESUMEN
Los cambios económicos, sociales, políticos y tecnológicos operados en estas tres últimas décadas, han permitido que la tecnología se constituya en un elemento esencial en las estrategias de las organizaciones para lograr la sobrevivencia, crecimiento y logro de los objetivos por ellas trazados, siendo necesario que sean productivas, que trabajen con calidad, competitividad y rentabilidad, dentro de un entorno local y global con la finalidad de que sean sostenibles en el tiempo.

La sociedad peruana está conformada por instituciones y organizaciones empresariales donde encontramos la presencia de tecnologías correspondientes a las civilizaciones agrícola, industrial y del conocimiento vigentes al mismo tiempo, por lo que muchas de estas unidades empresariales tienen niveles muy rudimentarios de aplicación de tecnologías de gestión, mientras que en otras organizaciones trabajan con un mayor nivel de modernidad.

El objeto del estudio de la investigación está orientado a la identificacion de las principales herramientas tecnológicas de gestión que concurren del plexo disciplinario de las ciencias administrativas en el ámbito de las organizaciones empresariales en Lima Metropolitana, así como identificar cuáles de ellas son de aplicación en las unidades empresariales. Al mismo tiempo se trata de indagar y desarrollar la descripción de sus paradigmas y formular propuestas que fortalezcan la incorporacion de herramientas tecnológicas en paradigmas que dinamicen y modernicen a las unidades empresariales, propendiendo a que su funcionamiento se realice con estándares de competividad de una dimensión global.
\end{abstract}

Palabras Clave: Herramientas tecnológicas, gestión, unidades empresariales, competitividad.

\begin{abstract}
The economic, social, political, technological operated these past three decades have allowed technology to become an essential element in the strategies of organizations, for their survival, growth and achievement of the objectives outlined for them, sustainable time, with productivity, quality, competitiveness and profitability within a local and global environment.
\end{abstract}

(*) Licenciado en cooperativismo. Docente Asociado de la Facultad de Ciencias Administrativas de la Universidad Nacional Mayor de San Marcos 
The Peru, as a society made up of business organizations, among others, the presence of technologies for the civilization of agriculture, industrial civilization and the civilization of knowledge, many of these business units with levels of technology application rudimentary or emerging management and other business units with higher levels of modernity.

The purpose of the research study will be aimed at the identification of key management tools that exist plexus administrative disciplinary science in the field of business organizations in metropolitan Lima, and which of them are applicable in the business units, investigating and developing the description of their paradigms and to make proposals to strengthen the incorporation into the application of technological tools embedded in paradigms that stimulate and modernize the business units tended to be accepted in its operating standards to compete on a global dimension.

Key Words: Technology tools, management, business unit, competitiveness.

\section{METODOS Y DISEÑO}

La metodología de investigación empleada para lograr la demostración y contrastación de las hipótesis es la identificación de las principales herramientas tecnológicas de gestión, y la descripción de los paradigmas en los que se sostienen en su aplicación actual las empresas en el espacio urbano de Lima Metropolitana. Además se formularán propuestas para fortalecer el uso de herramientas tecnológicas de gestión sustentadas en paradigmas modernos, que orienten a las empresas ser competitivas en un contexto global.

La investigación se concentra en la identificación de las principales tecnologías en el sector urbano de Lima Metropolitana. Se asume la clasificación de microempresas, pequeñas empresas, medianas y grandes empresas, con énfasis en la obtención de la información pertinente de las instituciones representativas del sector.

En el trabajo de campo se empleó el método de la encuesta estructurada en la realización de las entrevistas con los empresarios, gerentes y trabajadores, con el objeto de recabar información sobre el uso de las herramientas tecnológicas en las organizaciones empresariales. Así mismo, mediante las conversaciones realizadas, se captaron las percepciones referidas a las tecnologías de gestión y a los paradigmas que indujeron al empleo de las herramientas en los ámbitos de la organización y gestión en los siguientes segmentos empresariales: micro, pequeñas, medianas y grandes empresas.

\section{CONTRASTACION DE LA HIPÓTESIS}

\section{Hipótesis General}

Las tecnologías expresadas en sus herramientas de gestión incorporadas en la mayor parte de las unidades empresariales en el Perú se presentan en su aplicación como rudimentarias e incipientes, sostenidas por paradigmas en declive, por lo que se hace necesario que sean fortalecidas por otras herramientas tecnológicas de gestión sustentadas por paradigmas modernos, que orienten a las organizaciones empresariales para que sean competitivas dentro de una dimensión global.

\section{Contrastación de la Hipótesis}

Es indudable que hoy en día el propósito fundamental de las organizaciones empresariales que desean ubicarse en un contexto global es el de lograr eficiencia, eficacia, productividad, calidad y competitividad, siendo necesario la incorporación de tecnologías de gestión que les permitan realizar procesos dinámicos y de cambio, tal como impone el fenómeno de la globalización, del cual surgen los lineamientos de competitividad que tienen que desarrollar las empresas para tener mayores oportunidades de éxito en los negocios que emprenderán.

Los países desarrollados vienen haciendo frente al reto de la globalización y competitividad con cambios importantes en el uso de las tecnologías de gestión, con énfasis en la implantación de plataformas tecnológicas de información para los flujos de trabajo o procesos de las empresas, en la conformación de cadenas productivas, o impulsando que los clientes y proveedores se incorporen a las empresas como "colaboradores"; también impulsando cambios en los modelos organizacionales burocráticos y divisionales que muestran estructuras organizativas piramidales, para llegar a ser modelos organizacionales horizontales en red y virtuales.

Este propósito fundamental de las organizaciones empresariales peruanas se origina en la gran necesidad que tienen las micro, pequeñas, medianas y grandes empresas de adaptarse e inte- 
grarse a la variedad de cambios y de necesidades dentro de la diversidad cultural, que se derivan de las maneras o formas de llevar a cabo acciones que sustenten la competitividad de las empresas en los estadios de las tecnologías de la industrialización y de la era de la información y el conocimiento, utilizando sistemas, métodos, herramientas y procesos de trabajo conocidos como tecnologías de gestión que, desarrolladas de manera efectiva y con base a las necesidades de cada empresa y su fundamentación estratégica, permiten generar la competitividad empresarial.

Podemos definir a las tecnologías de gestión como el uso de las herramientas de gestión para tomar decisiones de manera racional, y para coordinar los talentos humanos y recursos disponibles para lograr resultados orientados a las necesidades de clientes y/o mercados; esto implica situar a las empresas como sistemas abiertos y ampliados, o sea conectado con su ambiente externo del que recibe influencias, considerando las interacciones principalmente entre el entorno, la estrategia, estructura, procesos, personal y productos y servicios que deseen obtener, procurando obtener ventajas competitivas que las distingan de la competencia, lo que implica desarrollar y transformar escenarios dentro del contexto de la globalización.

Para ser competitivas las empresas de nuestro entorno necesitan urgentemente reconfigurarse, considerando la información y tecnología para implantar e innovar procesos de cambio y mejora. En este contexto las micro, pequeñas y medianas empresas se han visto considerablemente afectadas por la extensión de la competitividad a escala mundial. En estos escenarios, mantener la cuota de mercado equivale a incrementar permanentemente la eficacia, eficiencia, productividad y calidad para que, de esa manera, se pueda dar respuesta a los requerimientos de los clientes y a la fuerte acción de la competencia.

Las micro, pequeñas y medianas empresas constituyen en el país, y particularmente el Lima, el grupo predominante; en algunos casos superan el $99 \%$ de unidades económicas y contribuyen significativamente a la creación de empleo, a la generación de riqueza y además satisfacen necesidades en ciertos mercados que son poco atractivos para las grandes empresas. Las micro, pequeñas y medianas empresas constituyen el grupo predominante en cualquier país, pero generalmente están articuladas con las empresas grandes a las cuales proveen de productos o servicios complementarios.

Las micro empresas en nuestro país tienen un alto índice de natalidad así como de mortandad, dado que carecen de suficientes capacidades y recursos, entre ellos los tecnológicos; no obstante para su sostenibilidad en el tiempo estas unidades empresariales podrían recurrir al mecanismo de la asociatividad mediante la forma empresarial de cooperativas de producción o de servicios. La asociatividad también tiene su expresión concreta en las cadenas productivas o cluster, alianzas estratégicas, join venture, o en la constitución formal de consorcios temporales; igualmente las formas empresariales de cooperativas pueden lograr su articulación formal mediante una central de cooperativas o las formas establecidas por la asociatividad.

También es de resaltar que las MYPEs, en su gran mayoría, no están en condiciones de asumir un costo fijo para contratar profesionales especializados en productividad y competitividad, por lo que es el gobierno peruano el que debe instituir políticas y estrategias que contribuyan a dar soporte tecnológico a estas unidades empresariales. Igualmente las universidades tienen un rol de aporte importante en la difusión de las tecnologías de gestión, así como en la innovación tecnológica.

Las micro, pequeñas y medianas empresas que lograron superar los desafíos de la supervivencia necesitan crecer y desarrollar ventajas competitivas, y ven que esto depende invariablemente de la competitividad que cada una genere a través de adecuados modelos de dirección y de la tecnología de gestión aplicados en el mercado internacional, nacional o local, en donde las empresas compiten entre sí, involucradas en los diversos sistemas productivos, esquemas institucionales y organizaciones sociales, en los que la empresa es un elemento importante. Para desarrollar ventajas competitivas es necesario efectuar procesos de gestión que -combinados con el talento humano, la infraestructura y relaciones, y aplicados de manera inteligente- permitirán gestionar el conocimiento creado dentro de una organización a través de metodologías que permitan realizar actividades de planeación, ejecución, evaluación y mejoramiento de forma secuencial, generando unidades empresariales con estándares internacionales de gestión. 
Las micro y pequeñas empresas presentan un sesgo con relación a la industrialización, según la Organización Internacional del Trabajo (OIT). En Lima, el 66\% responden al comercio, 26\% a los servicios y el $11 \%$ a la industria. Según el Ministerio de Trabajo y Promoción del Empleo (MTPE), la composición de las actividades de las PYMEs en Lima Metropolitana en 1998, fue la siguiente: el $44.5 \%$ pertenece al sector servicios, el $37 \%$ a comercio, industria el $18 \%$ y otros el $5 \%$. Es importante observar que una porción importante del las PYMEs se concentra en restaurantes y afines, así como en actividades relacionadas con el turismo. También se puede indicar que el mayor número de micro empresas se encuentran en la situación de informales.

Respecto a la descripción de las empresas en el Perú, particularmente en el área urbana de Lima Metropolitana, la Sociedad Nacional de Industrias (SNI) y la Cámara de Comercio de Lima (CCL), en el evento denominado "Hacia un Perú competitivo: oportunidades en tiempo de crisis" han afirmado que las tecnologías de gestión y específicamente la tecnología de la información y de las comunicaciones son un efectivo catalizador de la competitividad y de la búsqueda de oportunidades. Además consideran que, para estimular la adopción de las tecnologías de información y de las comunicaciones, se deben apuntalar las siguientes consideraciones:
- Tecnologías como prioridad clave en todos los paquetes de incentivo y estímulo que brinde el gobierno.

- Que el gobierno y el sector privado ejecuten programas de inversión en gran escala para el despliegue de la banda ancha en la educación, en el ámbito de las MYPEs y en las universidades, en programas de ciencia y tecnología.

En cuanto a las herramientas de gestión, en las siguientes líneas mostraremos su uso en el ámbito global según la información que proporciona el "Management Tools and Trends 2007", documento elaborado por Darrell Rigby y Barbara Bilodeau de Bain \& Company, que demuestra que hay una gran diversidad de tecnologías de gestión que son de aplicación en las unidades empresariales. En el Gráfico №1 se muestran las veinticinco herramientas técnicas más utilizadas actualmente en el ámbito mundial, según la investigación realizada por Bain \& Company.

Es de inferir que la rápida y correcta aplicación de estas herramientas en las unidades empresariales constituye un papel muy importante en el nivel de sobrevivencia, crecimiento, sostenibilidad y desarrollo de las mismas. Es por eso que es de suma importancia el conocimiento, uso y aplicación de estas herramientas tecnológicas y de otras para lograr crecimiento y desarrollo empresarial significativo, y así mantener a las

\section{Gráfico № 1}

Las herramientas de Tecnología de la Información más utilizadas por las empresas a nivel mundial PORCENTAJE DE USO DE TG

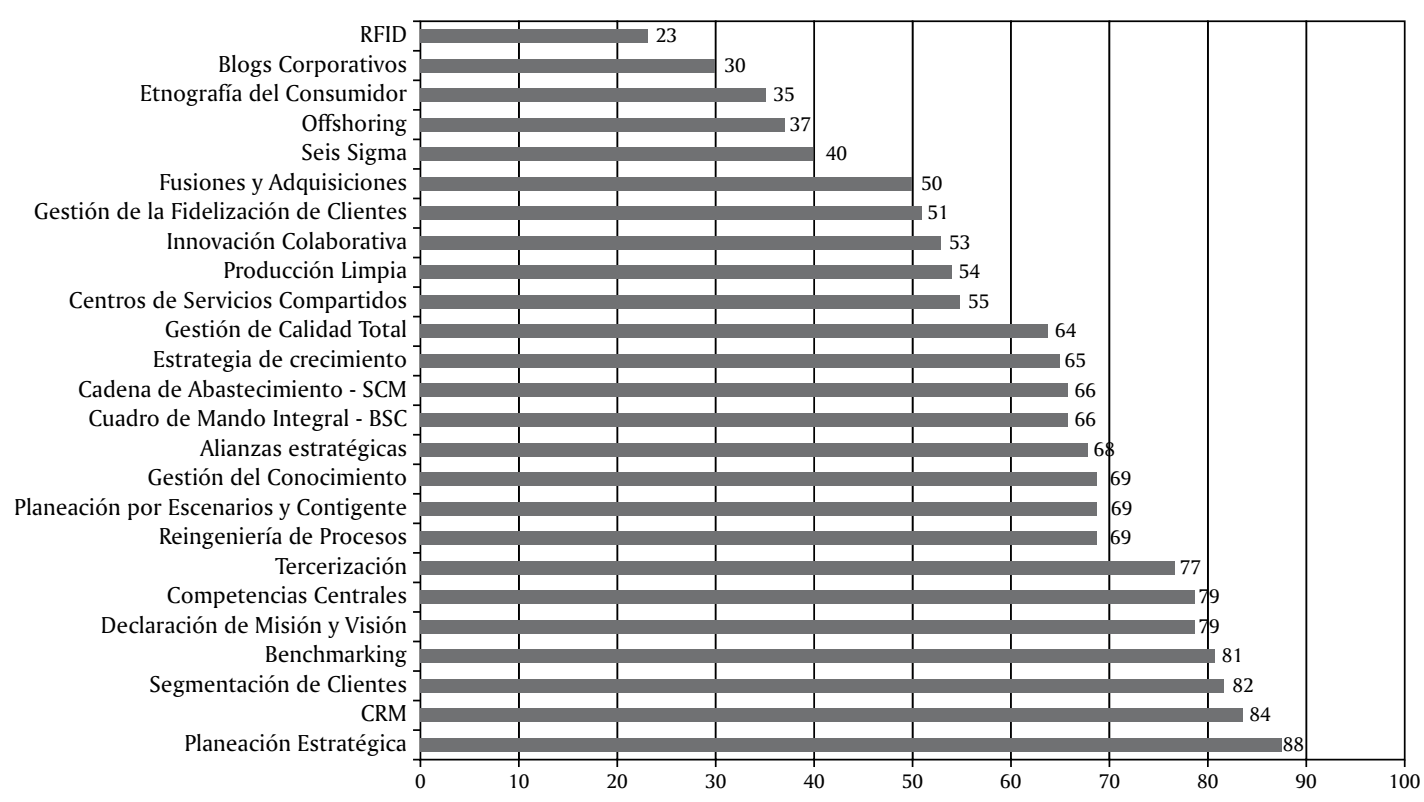


micro, pequeñas y medianas empresas dentro de las exigencias de los diferentes mercados en el marco de la globalización.

La importancia de investigar y cuestionar el tema de las tecnologías de gestión para la competitividad de las empresas, radica en que las unidades empresariales necesitan diagnosticar cuáles son sus necesidades; analizar la funcionalidad de las tecnologías que poseen actualmente y la posibilidad de combinar varias de ellas. Posteriormente, deben seleccionar las que consideren convenientes, para finalmente empezar a construir su propia tecnología de gestión y ser competitivas.

Las tecnologías de gestión no son nuevas hoy en día; podríamos decir que desde la revolución industrial se han desarrollado diversas y diferentes tecnologías de gestión, las mismas que han permitido que las empresas sean más productivas. Sin embargo, en la actualidad, las empresas no solo requieren ser productivas sino lograr competitividad, hecho derivado de la capacidad para conservar sistemáticamente las ventajas comparativas que les permitan conseguir, sustentar y optimizar una determinada posición en el entorno socioeconómico, hasta alcanzar ventajas competitivas sobre sus competidores. Es así que las tecnologías de gestión tendrán que aplicarse o desarrollarse con el objetivo de alcanzar esa capacidad, dentro de los estándares requeridos en cada contexto de interés dentro de la globalización.

En este trabajo mostraremos el uso de las herramientas de gestión en el ámbito de Lima Metropolitana, que es el resultado de las encuestas aplicadas en micro, pequeñas, medianas y grandes empresas como sigue:

- Micro empresas: 50 encuestas.

- Pequeñas y medianas empresas: 25 encuestas.

- Empresas grandes: 10 encuestas.

A continuación presentamos un listado de herramientas técnicas que provienen de las disciplinas que conforman el plexo de la ciencia administrativa, así como de su empleo en las micro, pequeñas, medianas y grandes empresas (ver Cuadro №1).

\section{Cuadro $\mathbf{N}^{\mathrm{o}} 1$}

Listado de herramientas técnicas y su empleo en las micro empresas

(Sobre 50 encuestas)

\begin{tabular}{|c|c|c|c|c|}
\hline \multirow[b]{2}{*}{$\begin{array}{c}\text { Campo del Proceso } \\
\text { de Gestión }\end{array}$} & \multirow[b]{2}{*}{$\begin{array}{l}\text { Herramientas } \\
\text { Técnicas }\end{array}$} & \multicolumn{3}{|c|}{ Empleo/Uso } \\
\hline & & $\begin{array}{c}\text { De uso } \\
\text { Tecnol. } \\
\text { Moderna }\end{array}$ & $\begin{array}{c}\text { De uso } \\
\text { Tecnol. } \\
\text { Rudimentaria }\end{array}$ & $\begin{array}{l}\text { No se } \\
\text { usa la herra- } \\
\text { mienta }\end{array}$ \\
\hline 1. Planeamiento & $\begin{array}{l}\text { - Planeamiento Estratégico } \\
\text { * Formulación de los principios y valores } \\
\text { *Formulación de Visión } \\
\text { *Formulación de la Misión } \\
\text { * Diagnóstico Estratégico } \\
\text { * Matrices Estratégicas: EFI, EFE, FODA, Matriz Competitiva, } \\
\text { Matriz BCG, Consistencia de Gestión, y Cuantitativa. } \\
\text { *Formulación de Objetivos Estratégicos } \\
\text { * Formulación de Metas Estratégicas } \\
\text { * Formulación de Políticas Estratégicas } \\
\text { - Planeamiento Táctico } \\
\text { *Formulación Planeamiento Táctico } \\
\text { *Evaluación Planeamiento Táctico } \\
\text { - Planeamiento Operativo } \\
\text { * Formulación de objetivos estratégicos } \\
\text { *Formulación de estrategias operativas } \\
\text { * Formulación del Plan de actividades } \\
\text { - Evaluación de la Visión } \\
\text { - Evaluación de la Misión } \\
\text { - Evaluación de los objetivos estratégicos y operativos } \\
\text { *Planeamiento de la calidad total } \\
\text { *Planeamiento de la reingeniería }\end{array}$ & & $\begin{array}{l}15 \\
03 \\
15 \\
15 \\
15 \\
03 \\
\\
15 \\
10 \\
04\end{array}$ & \\
\hline
\end{tabular}




\begin{tabular}{|c|c|c|c|c|}
\hline & $\begin{array}{l}\text { o ISO 9000-9001-14000 } \\
\text { o Elaboración de Proyectos } \\
\text { - Investigación de mercado } \\
\text { - Investigación motivacional } \\
\text { - Investigación de opinión } \\
\text { - Formulación de proyectos } \\
\text { - Evaluación de proyectos } \\
\text { Marco Lógico de Proyectos } \\
\text { - Presupuestos } \\
\text { - Evaluación de Ejecución Presupuestal } \\
\text { Benchmarking } \\
\text { - Estudio de Prefactibilidad técnico-económico } \\
\text { - Estudio de Factibilidad técnico-económico } \\
\text { - Perfil técnico-económico }\end{array}$ & & $\begin{array}{l}10 \\
10\end{array}$ & \\
\hline \multirow[b]{2}{*}{$\begin{array}{c}\text { Campo del Proceso } \\
\text { de Gestión }\end{array}$} & \multirow[b]{2}{*}{$\begin{array}{l}\text { Herramientas } \\
\text { Técnicas }\end{array}$} & \multicolumn{3}{|c|}{ Empleo/Uso } \\
\hline & & $\begin{array}{l}\text { De uso } \\
\text { moderno }\end{array}$ & $\begin{array}{c}\text { De uso } \\
\text { rudimentario }\end{array}$ & No se usa \\
\hline 2. Organización & $\begin{array}{l}\text { - Flujo de Caja } \\
\text { - Árboles de Decisión } \\
\text { - Teoría de los Juegos } \\
\text { - Teoría de las Colas } \\
\text { - Programación Lineal } \\
\text { - Pert-CPM } \\
\text { - Tormenta de ideas } \\
\text { - Diagrama de causa y efecto } \\
\text { - Diagrama de los } 5 \text { por qué } \\
\text { - Diagrama de afinidad } \\
\text { - Diagrama de Campos de fuerza } \\
\text { - Diagrama de Pareto 80-20 } \\
\text { - Estudio Costo/Beneficio } \\
\text { - Diagramas de flujo } \\
\text { - Diagrama de mallas } \\
\text { - Balance scorecard y tablero de control } \\
\text { - Diseños y Modelos organizacionales } \\
\text { - Análisis de puestos y/o cargos } \\
\text { - Clasificación de puestos y/o cargos } \\
\text { - Evaluación de puestos y/o cargos } \\
\text { - Benchmarking } \\
\text { - Racionalización administrativa } \\
\text { - Racionalización de procedimientos } \\
\text { - Reingeniería de procesos } \\
\text { - Calidad Total } \\
\text { - Las } 5 \text { S } \\
\text { - Sociodrama } \\
\text { - Evaluación de los grupos informales } \\
\text { - Evaluación del liderazgo } \\
\text { - Evaluación de las comunicaciones } \\
\text { - Planeamiento de los Recursos Humanos } \\
\text { - Reglamento de Organización y Funciones (ROF) } \\
\text { - Manual de Organización y funciones (MOF) } \\
\text { - Manual de Procedimientos } \\
\text { - Organigramas } \\
\text { - Diagramas de flujo } \\
\text { - Formulación de los estilos de dirección } \\
\text { - Evaluación de los estilos de dirección } \\
\text { Diona }\end{array}$ & & 15 & \\
\hline
\end{tabular}




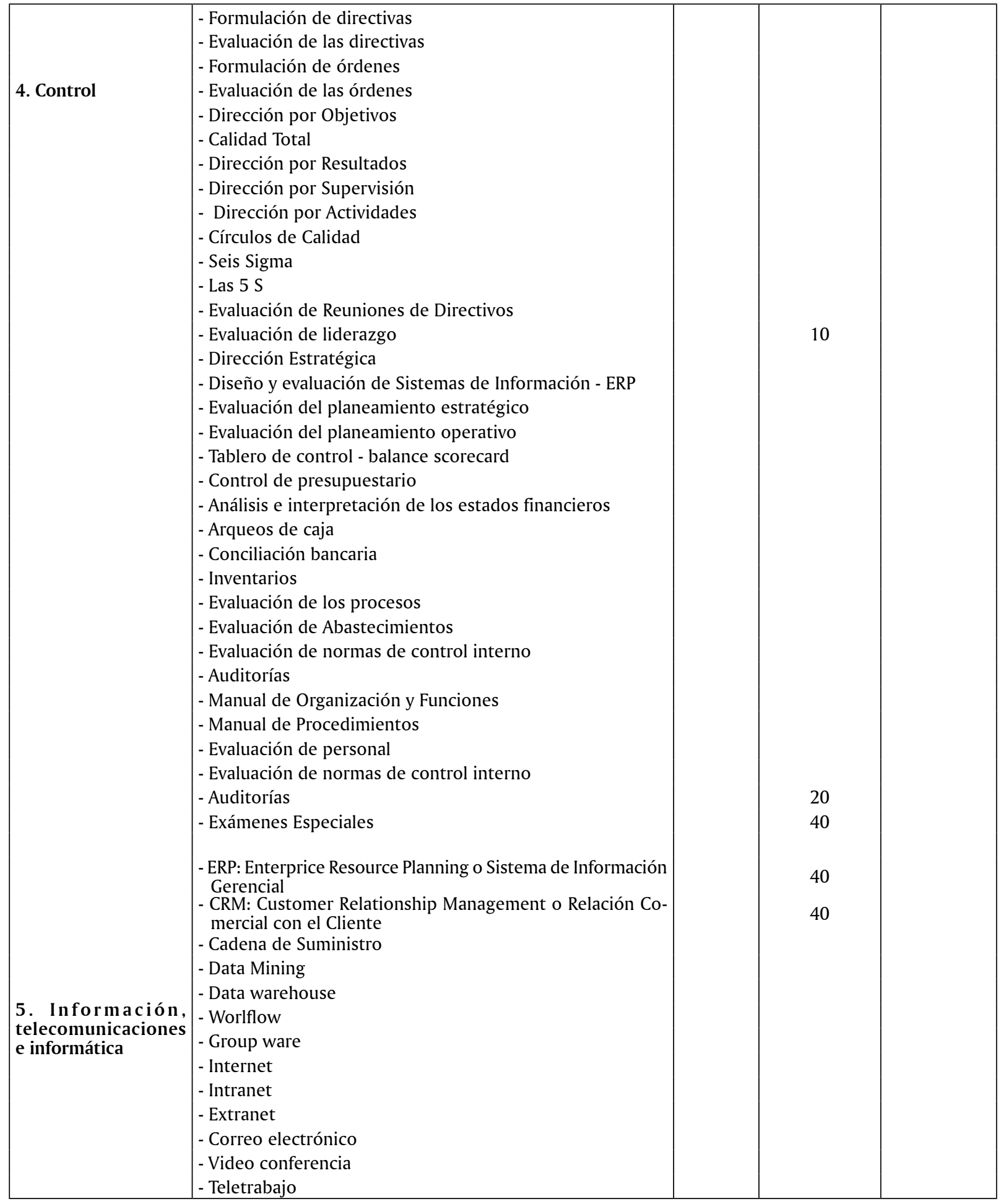

Es de verificarse que las micro empresas hacen uso de un número reducido de herramientas técnicas. Los porcentajes más significativos están representados por los arqueos de caja $(80 \%)$, control de inventarios $(80 \%)$, el análisis de los estados financieros $(20 \%)$ y en porcentajes menores el planeamiento estratégico $(30 \%)$ y la relación de costo-beneficio $30 \%$.
Es de observar el gran número de herramientas técnicas que no son empleadas por las micro empresas, lo que se debe posiblemente a la falta de difusión y de una insuficiente capacitación de las personas que conducen estas unidades empresariales.

Se ha podido observar el uso de las herramientas técnicas de forma rudimentaria, corres- 
pondiendo a un impulso práctico y empírico, extraordinariamente intuitivo, con una escasa teoría y sustentado en la práctica de sus conductores. Igualmente se observa cierto nivel de desconocimiento en la elaboración y en el uso de las herramientas, ya que las realizan fijando y privilegiando las ventas y los márgenes de utilidad con los que operan en el día a día.

Referente a los paradigmas de sus conductores, éstos se sostienen en el empleo de las herramientas tecnológicas, otorgando importancia y trascendencia a la experiencia obtenida por la práctica, al aprendizaje de los conocimientos $\mathrm{y}$ habilidades que han adquirido en el tiempo mediante la observación y practicas empíricas en otros centros de trabajo, con una experiencia promedio de tres a cuatro años. El $40 \%$ de los propietarios de micro empresas trabajan guiados por una adecuada teoría, primando su vocación por la aplicación práctica. Tienen predominancia los patrones de la civilización industrial, al exteriorizar en su voluntad y decisiones el permanecer con modelos organizativos tradicionales, dando preferencia a la estructura organizativa funcional en un $40 \%$ formalizada y en un $60 \%$ sin formalización, estructuras que se caracterizan por ser piramidales, jerarquizadas y centralizadas en la toma de decisiones.

Es de verificarse que en las pequeñas y medianas empresas el empleo consolidado de las herramientas técnicas de gestión presenta un mejor escenario que en las micro empresas, al hacer un mayor uso de las herramientas con mejores porcentajes, mostrando un $80 \%$ con el mayor número de las herramientas, en algunos el $60 \%$ y dirección por supervisión de un $100 \%$ (ver Cuadro №2).

\section{Cuadro $\mathrm{N}^{\circ} 2$}

Listado de herramientas técnicas y su empleo en las pequeñas y medianas empresas (Sobre 25 encuestas)

\begin{tabular}{|c|c|c|c|c|}
\hline \multirow[b]{2}{*}{$\begin{array}{c}\text { Campo del Proceso } \\
\text { de Gestión }\end{array}$} & \multirow[b]{2}{*}{$\begin{array}{l}\text { Herramientas } \\
\text { Técnicas }\end{array}$} & \multicolumn{3}{|c|}{ Empleo/Uso } \\
\hline & & $\begin{array}{c}\text { De uso } \\
\text { Tecnol. } \\
\text { Moderna }\end{array}$ & $\begin{array}{c}\text { De uso } \\
\text { Tecnol. } \\
\text { Rudimen- } \\
\text { taria }\end{array}$ & $\begin{array}{l}\text { No se } \\
\text { usa la herra- } \\
\text { mienta }\end{array}$ \\
\hline 1. Planeamiento & $\begin{array}{l}\text { - Planeamiento Estratégico } \\
\text { "Formulación de los principios y valores } \\
\text { "Formulación de Visión } \\
\text { "Formulación de la Misión } \\
\text { " Diagnóstico Estratégico } \\
\text { " Matrices Estratégicas: EFI, EFE, FODA, Matriz Competitiva, } \\
\text { Matriz BCG, Consistencia de Gestión, M. Cuantitativa. } \\
\text { "Formulación de Objetivos Estratégicos } \\
\text { "Formulación de Metas Estratégicas } \\
\text { "Formulación de Políticas Estratégicas } \\
\text { - Planeamiento Táctico } \\
\text { "Formulación Planeamiento Táctico } \\
\text { *Evaluación Planeamiento Táctico } \\
\text { - Planeamiento Operativo } \\
\text { "Formulación de objetivos estratégicos } \\
\text { "Formulación de estrategias operativas } \\
\text { "Formulación del Plan de actividades } \\
\text { - Evaluación de la Visión } \\
\text { - Evaluación de la Misión } \\
\text { - Evaluación de los objetivos estratégicos y operativos } \\
\text { * Planeamiento de la calidad total } \\
\text { "Planeamiento de la reingeniería } \\
\text { " ISO 9000-9001-14000 } \\
\text { o Elaboración de Proyectos } \\
\text { - Investigación de mercado } \\
\text { - Investigación motivacional } \\
\text { - Investigación de opinión } \\
\text { - Formulación de Proyectos } \\
\text { - Evaluación de Proyectos } \\
\text { Marco Lógico de Proyectos }\end{array}$ & & $\begin{array}{l}20 \\
20 \\
20 \\
20 \\
20 \\
20 \\
15\end{array}$ & \\
\hline
\end{tabular}




\begin{tabular}{|c|c|c|c|c|}
\hline & $\begin{array}{l}\text { - Presupuestos } \\
\text { - Evaluación de Ejecución Presupuestal } \\
\text { Benchmarking } \\
\text { - Estudio de Prefactibilidad técnico-económico } \\
\text { - Estudio de Factibilidad técnico-económico } \\
\text { - Perfil técnico-económico }\end{array}$ & & 20 & \\
\hline \multirow[b]{2}{*}{$\begin{array}{c}\text { Campo del Proceso } \\
\text { de Gestión }\end{array}$} & \multirow[b]{2}{*}{$\begin{array}{l}\text { Herramientas } \\
\text { Técnicas }\end{array}$} & \multicolumn{3}{|c|}{ Empleo/Uso } \\
\hline & & $\begin{array}{c}\text { De uso } \\
\text { moder- } \\
\text { no }\end{array}$ & $\begin{array}{c}\text { De uso } \\
\text { rudimen- } \\
\text { tario }\end{array}$ & No se usa \\
\hline 2. Organización & $\begin{array}{l}\text { - Flujo de Caja } \\
\text { - Árboles de Decisión } \\
\text { - Teoría de los Juegos } \\
\text { - Teoría de las Colas } \\
\text { - Programación Lineal } \\
\text { - Pert-CPM } \\
\text { - Tormento de ideas } \\
\text { - Diagrama de causa y efecto } \\
\text { - Diagrama de los } 5 \text { por qué } \\
\text { - Diagrama de afinidad } \\
\text { - Diagrama de Campos de fuerza } \\
\text { - Diagrama de Pareto 80-20 } \\
\text { - Estudio Costo/Beneficio } \\
\text { - Diagramas de flujo } \\
\text { - Diagrama de mallas } \\
\text { - Balance Scorecard y tablero de control } \\
\text { - Diseños y modelos organizacionales } \\
\text { - Análisis de puestos y/o cargos } \\
\text { - Clasificación de puestos y/o cargos } \\
\text { - Evaluación de puestos y/o cargos } \\
\text { - Benchmarking } \\
\text { - Racionalización administrativa } \\
\text { - Racionalización de procedimientos } \\
\text { - Reingeniería de procesos } \\
\text { - Calidad Total } \\
\text { - Las } 5 \text { S } \\
\text { - Sociodrama } \\
\text { - Evaluación de los grupos informales } \\
\text { - Evaluación del liderazgo } \\
\text { - Evaluación de las comunicaciones } \\
\text { - Planeamiento de los Recursos Humanos } \\
\text { - Reglamento de Organización y Funciones (ROF) } \\
\text { - Manual de Organización y funciones (MOF) } \\
\text { - Manual de Procedimientos } \\
\text { - Organigramas } \\
\text { - Diagramas de flujo } \\
\text { - Formulación de los estilos de dirección } \\
\text { - Evaluación de los estilos de dirección } \\
\text { - Formulación de directivas } \\
\text { - Evaluación de las directivas } \\
\text { - Formulación de órdenes } \\
\text { - Evaluación de las órdenes }\end{array}$ & & $\begin{array}{l}20 \\
20\end{array}$ & \\
\hline \multirow[b]{2}{*}{$\begin{array}{c}\text { Campo del Proceso } \\
\text { de Gestión }\end{array}$} & \multirow[b]{2}{*}{$\begin{array}{l}\text { Herramientas } \\
\text { Técnicas }\end{array}$} & \multicolumn{3}{|c|}{ Empleo/Uso } \\
\hline & & $\begin{array}{c}\text { De uso } \\
\text { moder- } \\
\text { no }\end{array}$ & $\begin{array}{c}\text { De uso } \\
\text { rudimen- } \\
\text { tario }\end{array}$ & No se usa \\
\hline 4.- Control & $\begin{array}{l}\text { - Dirección por objetivos } \\
\text { - Calidad total }\end{array}$ & & 25 & \\
\hline
\end{tabular}




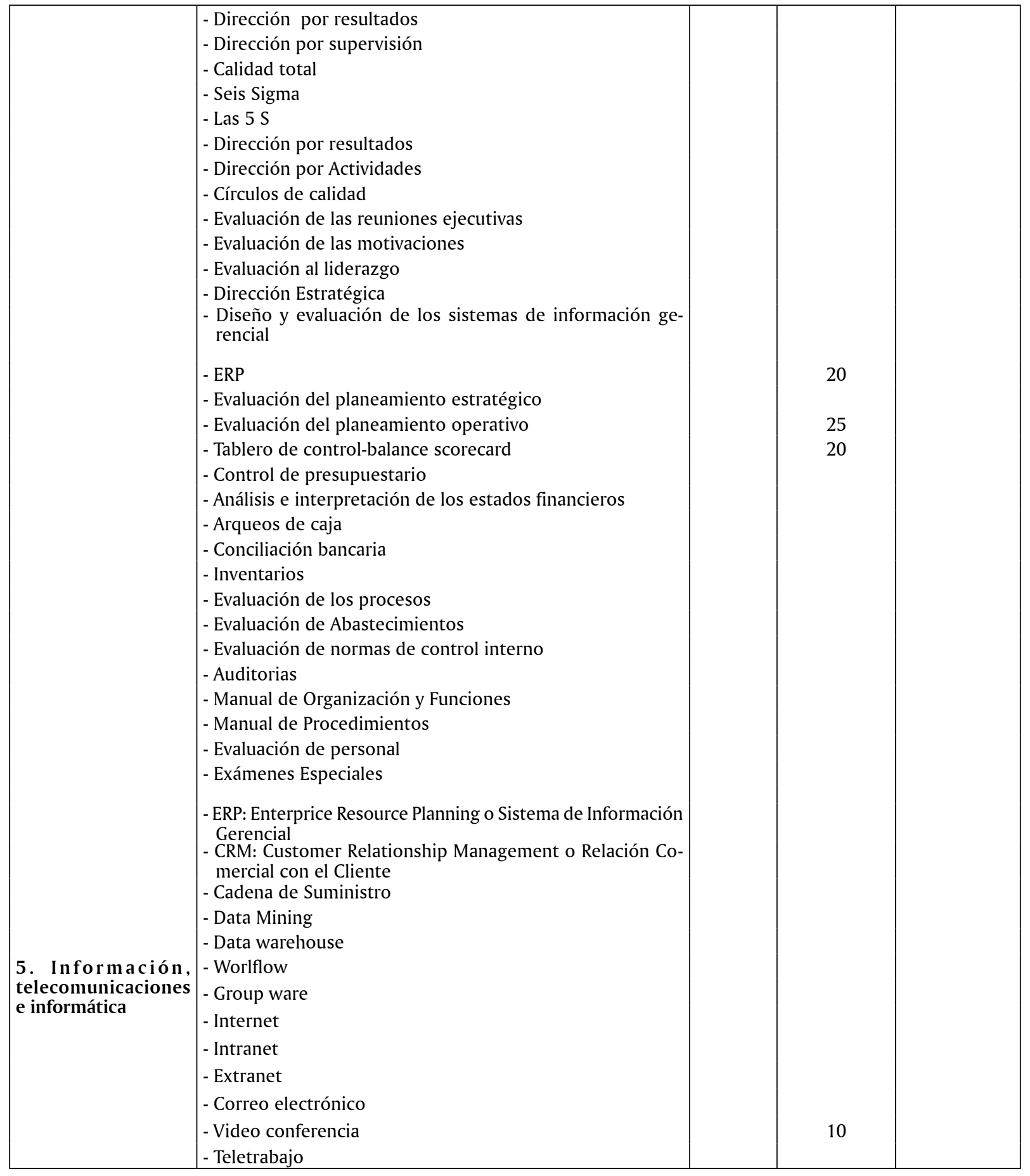

Es de observar el gran número de herramientas técnicas que no son empleadas en las pequeñas y medianas empresas, lo que posiblemente se debe a la falta de difusión de las mismas o a una insuficiente capacitación de las personas que conducen las medianas empresas; no obstante están en mejor nivel que los conductores de las micro empresas.

Se ha podido observar que el uso de las herramientas técnicas se efectúa siguiendo un impulso práctico y empírico en un $40 \%$, y a un esfuerzo teórico-práctico del $60 \%$ de los conductores de las medianas empresas. El $40 \%$ que las usa de forma rudimentaria o incipiente lo hace extraordinariamente, con una escasa teoría y sustentado en la práctica de sus conductores. Igualmente se observa un menor nivel de conocimiento que las micro empresas en la elaboración y en el uso de las herramientas. El $60 \%$ que usó las herramientas tiene mayor consistencia técnica. Ambos grupos implantan el uso de las herramientas fijando y 
privilegiando las ventas, los productos y servicios, la calidad y los márgenes de utilidad, así como el control con las que operan en el día a día, presentando mejores resultados el segmento del $60 \%$.

Referente a los paradigmas de sus conductores, un $60 \%$ se sostiene en el empleo de las herramientas tecnológicas, otorgando importancia y trascendencia al soporte teórico adecuado para su aplicación práctica. El aprendizaje de los conocimientos se ha realizado en centros superiores de enseñanza, complementados por una experiencia promedio de 3 a 4 años, y el $40 \%$ restante se sostiene en la experiencia práctica, reduciendo su vocación por una adecuada teoría. Pese a lo descrito aún tienen predominancia los patrones de la civilización industrial, al exteriorizar en su voluntad y decisiones el permanecer con modelos organizativos tradicionales, dando preferencia a la estructura organizativa funcional y en algunas de ellas utilizan una estructura mixta, incluyendo el modelo divisional en un $60 \%$ formalizada y un $40 \%$ sin formalizar.

Es de verificar que el mayor número de herramientas técnicas empleadas tienen el $100 \%$ de uso, no obstante hay un buen número de herramientas técnicas que no son empleadas por las empresas encuestadas.
Se ha podido observar el uso de las herramientas técnicas que corresponden a un impulso teórico-práctico de los conductores de empresas grandes y su nivel de elaboración y desarrollo de las herramientas responden a un nivel de estándar técnico satisfactorio del $90 \%$ de sus conductores (ver Cuadro № 3). Se implanta el uso de las herramientas a largo plazo, fijando estrategias que proyecten escenarios que privilegien la rentabilidad, la eficiencia operativa, el fortalecimiento de los ámbitos funcionales de las finanzas, operaciones y marketing con la finalidad de posicionarse competitivamente en el contexto local, e impulsando a que sus estándares tengan una adecuada posición estratégica en el contexto global.

Referente a los paradigmas de sus conductores, éstos se sostienen en el empleo de herramientas tecnológicas, otorgando importancia y trascendencia al soporte teórico adecuado para su aplicación práctica. El aprendizaje de los conocimientos se ha realizado en centros superiores de enseñanza, complementados por una experiencia promedio de tres a cuatro años; pese a lo descrito aún tienen predominancia los patrones de la civilización industrial, al exteriorizar en su voluntad y decisiones el permanecer con modelos organizativos tradicionales, dando preferencia a la estructura organizativa divisional.

\section{Cuadro $\mathbf{N}^{\circ} 3$}

Listado de herramientas técnicas y su empleo en las empresas grandes (Sobre 10 encuestas)

\begin{tabular}{|c|c|c|c|c|}
\hline \multirow{2}{*}{$\begin{array}{l}\text { Campo } \\
\text { del Proceso de } \\
\text { Gestión }\end{array}$} & \multirow[b]{2}{*}{$\begin{array}{l}\text { Herramientas } \\
\text { Técnicas }\end{array}$} & \multicolumn{3}{|c|}{ Empleo/Uso } \\
\hline & & $\begin{array}{l}\text { De uso } \\
\text { Tecnol. } \\
\text { Moderna }\end{array}$ & $\begin{array}{c}\text { De uso } \\
\text { Tecnol. } \\
\text { Rudimentaria }\end{array}$ & $\begin{array}{l}\text { No se } \\
\text { usa la he- } \\
\text { rramienta }\end{array}$ \\
\hline 1. Planeamiento & $\begin{array}{l}\text { - Planeamiento Estratégico } \\
\text { "Formulación de los principios y valores } \\
\text { "Formulación de Visión } \\
\text { "Formulación de la Misión } \\
\text { "Diagnóstico Estratégico } \\
\text { " Matrices Estratégicas: EFI, EFE, FODA, Matriz Competitiva, } \\
\text { Matriz BCG, Consistencia de Gestión, M. Cuantitativa. } \\
\text { "Formulación de Objetivos Estratégicos } \\
\text { "Formulación de Metas Estratégicas } \\
\text { "Formulación de Políticas Estratégicas } \\
\text { - Planeamiento Táctico } \\
\text { "Formulación Planeamiento Táctico } \\
\text { "Evaluación Planeamiento Táctico } \\
\text { - Planeamiento Operativo } \\
\text { "Formulación de objetivos estratégicos } \\
\text { * Formulación de estrategias operativas } \\
\text { "Formulación del Plan de Actividades } \\
\text { - Evaluación de la Visión } \\
\text { - Evaluación de la Misión } \\
\text { - Evaluación de los objetivos estratégicos y operativos }\end{array}$ & & $\begin{array}{l}10 \\
10 \\
10 \\
10 \\
\\
10 \\
10 \\
10\end{array}$ & \\
\hline
\end{tabular}




\begin{tabular}{|c|c|c|c|c|}
\hline & $\begin{array}{l}\text { * Planeamiento de la calidad total } \\
\text { * Planeamiento de la reingeniería } \\
\text { * ISO 9000-9001-14000 } \\
\text { o Elaboración de Proyectos } \\
\text { - Investigación de mercado } \\
\text { - Investigación motivacional } \\
\text { - Investigación de opinión } \\
\text { - Formulación de Proyectos } \\
\text { - Evaluación de Proyectos } \\
\text { Marco Lógico de Proyectos } \\
\text { - Presupuestos } \\
\text { - Evaluación de Ejecución Presupuestal } \\
\text { Benchmarking } \\
\text { - Estudio de Prefactibilidad técnico-económico } \\
\text { - Estudio de Factibilidad técnico-económico } \\
\text { - Perfil técnico-económico } \\
\end{array}$ & & $\begin{array}{l}10 \\
10 \\
10 \\
10 \\
05 \\
\\
10 \\
10 \\
\\
10 \\
10\end{array}$ & \\
\hline \multirow{2}{*}{$\begin{array}{l}\text { Campo } \\
\text { Funcional }\end{array}$} & \multirow{2}{*}{$\begin{array}{l}\text { Herramientas } \\
\text { Técnicas }\end{array}$} & \multicolumn{3}{|c|}{ Empleo/Uso } \\
\hline & & $\begin{array}{l}\text { De uso } \\
\text { moderno }\end{array}$ & $\begin{array}{c}\text { De uso } \\
\text { rudimentario }\end{array}$ & No se usa \\
\hline 2.Organización & $\begin{array}{l}\text { - Flujo de Caja } \\
\text { - Árboles de Decisión } \\
\text { - Teoría de los Juegos } \\
\text { - Teoría de las Colas } \\
\text { - Programación Lineal } \\
\text { - Pert-CPM } \\
\text { - Tormento de ideas } \\
\text { - Diagrama de causa y efecto } \\
\text { - Diagrama de los } 5 \text { por qué } \\
\text { - Diagrama de afinidad } \\
\text { - Diagrama de Campos de fuerza } \\
\text { - Diagrama de Pareto 80-20 } \\
\text { - Estudio Costo/Beneficio } \\
\text { - Diagramas de flujo } \\
\text { - Diagrama de mallas } \\
\text { - Balance scorecard y tablero de control } \\
\text { - Diseños y Modelos organizacionales } \\
\text { - Análisis de puestos y/o cargos } \\
\text { - Clasificación de puestos y/o cargos } \\
\text { - Evaluación de puestos y/o cargos } \\
\text { - Benchmarking } \\
\text { - Racionalización administrativa } \\
\text { - Racionalización de procedimientos } \\
\text { - Reingeniería de procesos } \\
\text { - Calidad Total } \\
\text { - Las } 5 \text { S } \\
\text { - Sociodrama } \\
\text { - Evaluación de los grupos informales } \\
\text { - Evaluación del liderazgo } \\
\text { - Evaluación de las comunicaciones } \\
\text { - Planeamiento de los Recursos Humanos } \\
\text { - Reglamento de Organización y Funciones (ROF) } \\
\text { - Manual de Organización y Funciones (MOF) } \\
\text { - Manual de Procedimientos } \\
\text { - Organigramas } \\
\text { - Diagramas de flujo } \\
\text { - Dino }\end{array}$ & & & \\
\hline
\end{tabular}




\begin{tabular}{|c|c|c|c|c|}
\hline 3. Dirección & $\begin{array}{l}\text { - Formulación de los estilos de dirección } \\
\text { - Evaluación de los estilos de dirección } \\
\text { - Formulación de directivas } \\
\text { - Evaluación de las directivas } \\
\text { - Formulación de órdenes } \\
\text { - Evaluación de las órdenes }\end{array}$ & & & \\
\hline \multirow[b]{2}{*}{$\begin{array}{l}\text { Campo } \\
\text { Funcional }\end{array}$} & \multirow[b]{2}{*}{$\begin{array}{l}\text { Herramientas } \\
\text { Técnicas }\end{array}$} & \multicolumn{3}{|c|}{ Empleo/Uso } \\
\hline & & $\begin{array}{l}\text { De uso } \\
\text { moderno }\end{array}$ & $\begin{array}{c}\text { De uso } \\
\text { rudimentario }\end{array}$ & No se usa \\
\hline $\begin{array}{l}\text { 5. In formación, } \\
\text { telecomunicaciones } \\
\text { e informática }\end{array}$ & 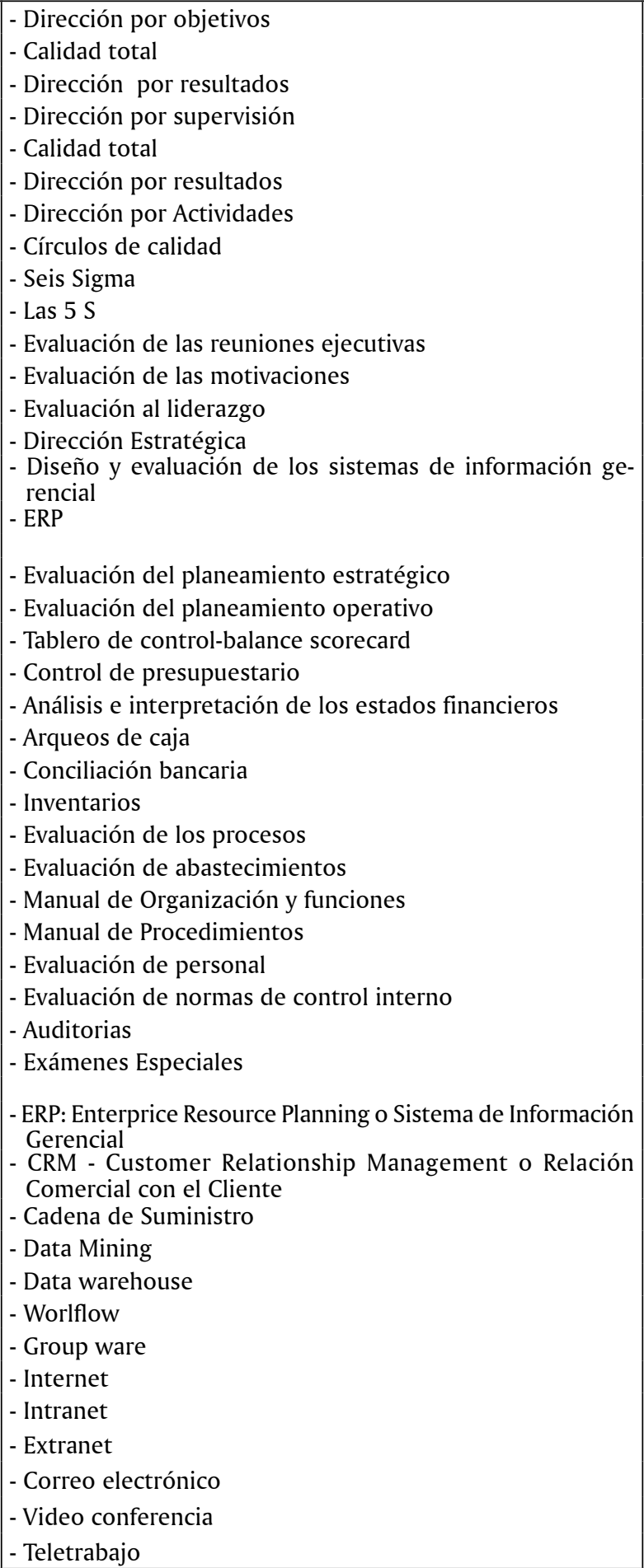 & & & \\
\hline
\end{tabular}




\section{CONCLUSIONES}

1. Las herramientas de gestión incorporadas en la mayor parte de las micro y pequeñas empresas en Lima Metropolitana se presentan en su aplicación como rudimentarias e incipientes, sostenidas por paradigmas sustentados en privilegiar la práctica y el empirismo en relacion con la teoría. Los avances tecnológicos los ubican en una tendencia de declinacion.

2. Las herramientas de gestión aplicadas en la mediana y la gran empresa en Lima Metropolitana muestran una tendencia a situarse al ritmo de los avances tecnológicos; no obstante el "efecto paradigma" actúa como un factor retardatario.

3. No obstante la situación del escenario empresarial, las empresas están motivadas para ser más eficientes, eficaces y competitivas dentro del ámbito nacional y con una dimension global, por lo que demandan ser fortalecidas por la tecnificación en gestión y sustentadas por paradigmas modernos.

\section{RECOMENDACIONES}

Las recomendaciones que fluyen de la investigacion se expresan como sigue:

- Promover y difundir las tecnologías de gestión y sus herramientas para que se constituyan en un elemento esencial en las estrategias de las organizaciones, que permitan su sobrevivencia, crecimiento y logro de sus objetivos, permitiendo que sean sostenibles en el tiempo, acompañadas de productividad, calidad, competividad y rentabilidad dentro de un entorno local y global.

- Promover y difundir el empleo de las herramientas tecnológicas que otorgan importancia y trascendencia, así como el soporte teórico adecuado para su aplicación práctica. También requieren del aprendizaje de los conocimientos, correspondiendo a las universidades y otros centros superiores un rol de capital relevancia en la adopción, adecuación e innovación de las herramientas tecnológicas, así como su promoción y difusión para una buena práctica en las unidades empresariales.

- Correspondería al Estado peruano brindar un mayor apoyo a las universidades públicas para que estas implanten efectivos centros de tecnología e innovación.

\section{BIBLIOGRAFÍA}

Andriani, C.; Biasca, R.; Rodríguez, M. (2003). El nuevo sistema de Gestión para las Pymes. México, Editorial Norma.

Anssoff Igor H. (1996). Dirección Estratégica. Conceptos, técnicas y aplicaciones. Madrid, Editorial Civitas.

B. J. Hogde, WP. Anthony, L.M. Gales. (1999). Teoría de la Organización:Un Enfoque Estratégico. México, Quinta Edición Prentice Hall.

Bill Gates. (1995). Camino al Futuro. España, Editores Plaza James S.A.

David, Fred R. (1989). Administración Estratégica. 5ta. Edición. Mejico, Prentice Hall

Don Tapscott y Art Caston. (1995). Cambio de paradigmas empresariales. México, McGraw Hill.

Espinoza Herrera, Nemesio. (2008). Gestión de Pymes. Lima, Editorial Imprenta de San Marcos.

Hammer, Michael y Champy, James. Reingeniería. (1998). Bogotá. Editorial Norma.

Hodge, B.J. Anthony, W.P. Gales, L.M. (1998). Teoría de la Organización. Madrid, Prentice Hall Iberia, S.R.L.

Huaco Pastor, Enrique. (2005). Guía Técnica para el Diseño Organizacional. Lima, Editorial San Marcos.

José Luis Lossio. (2005). Elementos del Diseño Organizacional. Segunda Edición. Lima, Grafica Interamericana s.a.c.

Kast E., Fremont y Rosenzweing, James E. (1994). Enfoque de Sistemas y Contingencias. 4ta. Edición. México, Mc Graw-Hill.

Mintzherg Henrry. (2000). Estructura Organizacional. Barcelona, Editorial Ariel S.A.

Mintzherg, Henry. (1997). El Proceso Estratégico. Barcelona, Editorial Prentice Hall.

Oster, S. (2000). Análisis Moderno de la Competitividad. México, Oxford.

Peppard y Rowland. (1997). La Esencia de la Reingeniería en los Procesos de Negocios. México, Prentice Hall Hispanoamericana.

Richar L. Daft. (2005). Teoría y Diseño Organizacional. Octava Edición. México, Thomson. 
Serna Gómez, Guillermo. (1992). Planeación y Organización de Empresas. 6ta. Edición. Bogotá, Prentice Hall.

Tapscott, Don. (1995). Ganar en la Economía Digital. México, Mc Graw Hill. 